\title{
TOTAL FLAVONOIDS CONTENT OF SOME SEASONAL FRUITS AND THEIR BENEFICIAL EFFECTS ON CARDIOVASCULAR DISEASE: A CROSS-SECTIONAL STUDY
}

\author{
SARA NAQVI ${ }^{1}$, SHAHLLA IMAM ${ }^{1,2 *}$, FATIMA RAMZAN ALI ${ }^{3}$, IQBAL AZHAR ${ }^{1}$, ZAFAR ALAM MAHMOOD ${ }^{1}$ \\ ${ }^{1}$ Department of Pharmacognosy, Faculty of Pharmacy and Pharmaceutical Sciences, University of Karachi, Karachi, Pakistan. ${ }^{2}$ Department \\ of Pharmacognosy, Institute of Pharmaceutical Sciences, Jinnah Sindh Medical University, Karachi, Pakistan. ${ }^{3}$ Department of Pharmaceutics, \\ Faculty of Pharmacy and Pharmaceutical Sciences, University of Karachi, Karachi, Pakistan. Email: shahlla.imam@jsmu.edu.pk
}

Received: 28 March 2020, Revised and Accepted: 08 May 2020

ABSTRACT

Objective: The present study was intended to assess the qualitative and quantitative analysis of some fruits available in Karachi, Pakistan. The association between the intakes of flavonoids enriches fruit and cardiovascular (CV) health was evaluated through a clinical survey.

Methods: The acid-alkaline test was executed in all fruit samples. The total flavonoids content was carried out using aluminum chloride calorimetric method. A cross-sectional survey was executed from July to December 2018 among 100 cardiac patients in Karachi, Pakistan. The patient's blood pressure was determined and categorized into normal, prehypertension, and hypertension. Descriptive statistics and Pearson's Chi-squared test were executed to demonstrate the participants' sociodemographic information.

Results: The result revealed that the purple grapes contained an abundant amount of total flavonoid contents $192.59 \pm 1.28$ as compared to green grapes and other fruits, whereas Citrus paradisi (grapefruit), Citrus reticulata (kinnow), and Punica granatum (pomegranate) contained $162.96 \pm 0.640$, $151.85 \pm 0.640$, and 107.03 \pm 1.28 , respectively. Moreover, Malus species, grapes (green), raspberry, and strawberry are also a good source of flavonoids. The clinical survey revealed that the majority of the respondents were male $74 \%$ while only $26 \%$ were female. The response rate was $73 \%$.

Conclusion: The finding of the present study indicated that dietary intake of fruits containing flavonoids may decrease the risk of CV disease and could contribute to promoting cardiac health.

Keywords: Cardioprotective, Cardiovascular risk, Cross-sectional survey, Fruits, Total flavonoids content, Hypertension.

(C) 2020 The Authors. Published by Innovare Academic Sciences Pvt Ltd. This is an open access article under the CC BY license (http://creativecommons. org/licenses/by/4. 0/) DOI: http://dx.doi.org/10.22159/ajpcr.2020.v13i7.37782

\section{INTRODUCTION}

All over the world, cardiovascular diseases (CVDs) are the major cause of mortality. It remains the primary cause of death and strongly associated with complications with increased morbidity. Several risk factors for the pathogenesis of CVD are hypertension, hyperlipidemia, endothelial dysfunction, smoking, obesity, diabetes mellitus, unhealthy diet, and sluggish lifestyle [1].

Diet plays a major role to maintain a healthy lifestyle, especially in the prevention of metabolic and chronic diseases. The phytochemicals are an essential part of the food that has numerous health promising effects. Epidemiological studies have been demonstrated that consumption of diet enriched with polyphenols such as flavonoids, phenolic acids, carotene anthocyanin, stilbenes, and lignans decreased the risk of diabetes, osteoporosis, cancer, and CVD [2,3]. Flavonoids are heterocyclic polyphenolic compounds extensively disseminated in the plant kingdom and classified into subgroups based on their chemical structure, that is, flavonols, flavones, isoflavones, flavanones, flavan-3-ols, anthocyanidins, and proanthocyanidins. Flavonoids are found in a variety of plant-derived foods including fruits (grape, oranges, mandarin, apple, cherry, strawberry, and blueberry), tea (green and black), coffee, spices, vegetables, especially cruciferous, green leafy vegetables (spinach, lettuce, onion, celery, parsley, broccoli, and bean), legumes herbs, and grains $[4,5]$.

Flavonoids contribute to the utmost part of the human diet have an integral role in preventing the occurrence of CVD complications. The promising health benefits of flavonoids are due to their metal ion chelating and antioxidant properties. Several biological activities of flavonoids, that is, hepatoprotective, antimicrobial, antidiabetic, antiinflammatory, antithrombotic, antihyperlipidemic, anticancer, and cardioprotective properties have been reported [6]. Consequently, the present study was intended to evaluate the flavonoid contents in some fruits available in Karachi, Pakistan, and assessed the association between the intakes of flavonoids enrich fruits on CVDs.

\section{METHODS}

\section{Plant materials}

The ripe fruit samples comprised papaya, grapefruit, Citrus fruit (kinnow, fruiter, mosami, and malta), strawberry, sapodilla, apple (golden and kulu), pomegranate, raspberry, and grapes (green and purple) were procured from the local market of Karachi, Pakistan, during their season. The fruit samples were authenticated from the Department of Pharmacognosy, Faculty of Pharmacy and Pharmaceutical Sciences, University of Karachi, Karachi, Pakistan.

\section{Preparation of extracts}

Fruit samples ( $1 \mathrm{~kg}$ each sample) were washed thoroughly to remove dirt and irrelevant material then cut into small pieces. It was then macerated separately in a mixture of a solvent comprising of methanol, ethanol $(35 \%)$, and distilled water (30\%) for 8-10 days at room temperature. The contents were filtered and concentrated under reduced pressure using a rotary evaporator. The crude hydroalcoholic fruit extract was obtained from each fruit. It was then stored in a well-closed container and kept in a refrigerator $\left(4^{\circ} \mathrm{C}\right)$ for further analysis.

\section{The acid-alkaline test}

An acid-alkaline test was executed to confirm the presence of flavonoids in all crude fruit extracts. Approx. $3 \mathrm{ml}$ of each fruit extract was treated separately with a few drops of sodium hydroxide solution $(2 \%)$. An intense yellow color precipitate was formed. It was then turned into a colorless solution on addition of $0.5 \mathrm{ml}$ of concentrated hydrochloric acid indicated the presence of flavonoids in each fruit sample. 
Table 1: Qualitative and quantitative evaluation of some seasonal fruits

\begin{tabular}{|c|c|c|c|}
\hline Scientific name & English name & Flavonoids test & TFC \\
\hline V. vinifera & Grapes (purple) & ++++ & $192.59 \pm 1.28$ \\
\hline Citrus paradisi & Grape fruit & ++++ & $162.96 \pm 0.64$ \\
\hline Citrus reticulata & Mandarin orange (kinnow) & ++++ & $151.85 \pm 0.64$ \\
\hline Punica granatum & Pomegranate (kandhari) & +++ & $107.03 \pm 1.28$ \\
\hline Citrus reticulata & Blanco Marisol (fruiter) & +++ & $97.14 \pm 0.57$ \\
\hline V. vinifera & Grapes (green) & +++ & $86.28 \pm 0.646$ \\
\hline Citrus sinensis & Maltaise Sanguine (Malta) & ++ & $69.99 \pm 1.11$ \\
\hline Citrus sinensis & Valencia (mosami) & ++ & $61.85 \pm 0.64$ \\
\hline Rubus idaeus & Raspberry & ++ & $59.62 \pm 0.64$ \\
\hline Fragaria ananassa & Strawberry & ++ & $58.14 \pm 0.64$ \\
\hline Malus domestica & Apple (golden) & ++ & $45.92 \pm 0.64$ \\
\hline Carica papaya & Papaya & + & $36.3 \pm 0.64$ \\
\hline Manilkara zapota & Sapodilla (round) & + & $27.77 \pm 1.11$ \\
\hline
\end{tabular}

Data are presented as mean \pm SD; ++++: Excellent amount; +++: Good; ++: Moderate; +: Trace amount. V. vinifera: Vitis vinifera

\section{Determination of total flavonoid content}

Aluminum chloride $\left(\mathrm{AlCl}_{3}\right)$ calorimetric method was performed to determine the total flavonoid content in all crude extracts $[7,8]$. Approximately $0.5 \mathrm{ml}$ of fruit extract solution was added in $1.5 \mathrm{ml}$ of methanol (95\%). About $0.1 \mathrm{ml}$ of $\mathrm{AlCl}_{3}(10 \%)$ and $0.1 \mathrm{ml}$ of potassium acetate $(1 \mathrm{M})$ and $2.8 \mathrm{ml}$ of distilled water were added in a methanolic solution of fruit sample. The absorbance of the solution was noted at $415 \mathrm{~nm}$. Blank was prepared by substituting $\mathrm{AlCl}_{3}$ with distilled water. The contents of total flavonoid were recorded using the quercetin standard $(\mathrm{QE} \mu \mathrm{g} / \mathrm{g})$ calibration curve $\left(\mathrm{y}=0.0009 \mathrm{x}+0.001, \mathrm{r}^{2}=0.9189\right)$. The same procedure was repeated with all fruit samples.

\section{Clinical study}

Study protocol

A cross-sectional study was accomplished from July to December 2018. The study population consisted of 73 cardiac patients from public and private sector hospitals around Karachi, Pakistan, were approached to assess their knowledge and attitudes toward flavonoids enriched diet. The questionnaire was distributed to the individuals who participated in the study. The study participants were divided into three groups; normotensive (blood pressure $=120 / 80 \mathrm{mmHg}$ ), prehypertensive group (120-139/80-89 $\mathrm{mmHg}$ ), and hypertensive group ( $\geq 140 / 90 \mathrm{mmHg}$ ) [9]. Each group consumption of fruit as a part of a normal healthy diet was assessed. The literature review was carried out to develop the questionnaire and compile information about flavonoids enriched diet as cardioprotective. The search engine Embase, Medline, PubMed, and Web of Science database has been used for literature review. Some information was given to the participants and it was kept strictly confidential.

\section{Inclusion criteria}

All participants with aged groups ranging from 40 to 75 years of age with or without hypertension from both genders were included in the study.

\section{Exclusion criteria}

Patients with a family history of diabetes mellitus, hyperlipidemia, and blood disorders were excluded from the study. The period and frequency of consumption of dietary flavonoids were also excluded from the study.

\section{Data analysis}

Statistical Package for the Social Sciences (SPSS version 20; SPSS Inc., Chicago, IL, USA) was executed for analysis. Pearson's Chi-square test $(p<0.05)$ was applied to assess the association between sociodemographic features and questions regarding the consumption of fruit stuff. Gender, age group, and medicines prescribed for other disease were independent variables. The results were depicted in terms of means, frequencies, and percentages.
Table 2: Demographic data of the study participants

\begin{tabular}{ll}
\hline Variables & Frequency (\%) \\
\hline Gender & \\
Male & $54(74)$ \\
Female & $19(26)$ \\
Age groups (years) & \\
41-48 & $8(11)$ \\
$49-56$ & $21(28.8)$ \\
$57-64$ & $23(31.5)$ \\
65-72 & $21(28.8)$ \\
B.P. group & \\
Normal B.P. (80-120) & $8(11)$ \\
Prehypertension $(>80-139)$ & $28(38.4)$ \\
Hypertension $(>90-140)$ & $37(50.7)$ \\
\hline
\end{tabular}

\section{RESULTS AND DISCUSSION}

Despite great advancement within the field of synthetic drugs, plants still have their unique position, due to numerous health benefits and fewer side effects. Several research studies recommended that nutritional pattern has been directly linked with the prevention of chronic diseases for instance; cancer, diabetes, Alzheimer, renal, liver, and heart diseases [4]. In the present study, qualitative and quantitative analysis of some fruits was executed to evaluate the total flavonoid contents. The acid-alkaline test illustrated in Table 1 confirmed the presence of flavonoid in all fruit samples.

$\mathrm{AlCl}_{3}$ is one of the most widely used method, applied to the determination of total flavonoid content in various plants and herbs [7]. The result revealed that all fruit sample contained good, moderate, and an appreciable amount of flavonoids. Vitis vinifera (purple grapes) showed the excellent amount of flavonoid content, that is, 192.59 \pm 1.28 and could be considered as the richest source of flavonoid among the selected fruits, whereas Citrus paradisi (grapefruit) and Citrus reticulata (kinnow) also have a substantial amount of flavonoid contents. In addition, Punica granatum (pomegranate), C. reticulata Blanco Marisol (fruiter), and V. vinifera (green grapes) were a good source of flavonoid. While a moderate amount of total flavonoids content was noted in Citrus species (malta and mosami), apple (golden and kulu), raspberry, and strawberry, whereas the papaya and sapodilla contained a small amount of flavonoid content. The contents of flavonoid in fruit samples are illustrated in Table 1. Pakistan is an agricultural country and has immense potential to produce numerous fruits with several varieties. Papaya, grapefruit, Citrus fruits (kinnow, fruiter, mosami, and malta), pomegranate, sapodilla, apple (golden and kulu), strawberry, and grapes (green and purple) are extensively distributed in different areas of Pakistan. However, the distribution of flavonoids content is varying due to various factors including endogenous factor, geographical region, climatic factor, cultivation, harvesting technique, ripening, 
Table 3: Consumption of fruits and its impact on blood pressure

\begin{tabular}{|c|c|c|c|c|c|}
\hline \multirow[t]{2}{*}{ Fruits } & \multirow{2}{*}{$\begin{array}{l}\text { Normotensives } \\
\text { n (\%) }\end{array}$} & \multirow{2}{*}{$\begin{array}{l}\text { Prehypertension } \\
\mathbf{n}(\%)\end{array}$} & \multirow{2}{*}{$\begin{array}{l}\text { Hypertension } \\
\mathbf{n}(\%)\end{array}$} & \multirow{2}{*}{$\begin{array}{l}\text { Total Consumption } \\
\text { n (\%) }\end{array}$} & \multirow[t]{2}{*}{ p-value } \\
\hline & & & & & \\
\hline Apple & $8(11)$ & $28(38.4)$ & $25(34.2)$ & $61(83.6)$ & $0.001^{* *}$ \\
\hline Banana & $3(4.1)$ & $26(35.6)$ & 18 (24.7) & $47(64.4)$ & $0.000 * * *$ \\
\hline Orange & $8(11$ & $27(37$ & $23(31.5$ & $58(79.5$ & $0.001^{* *}$ \\
\hline Watermelon & $3(4.1)$ & $19(26)$ & 16 (21.9) & 38 (52.1) & 0.099 \\
\hline Grapefruit & $6(8.2)$ & 17 (23.3) & 13 (17.8) & $36(49.3)$ & $0.038^{*}$ \\
\hline Peach & $4(5.5)$ & $13(17.8)$ & $8(11)$ & $25(34.2)$ & 0.069 \\
\hline Strawberry & $5(6.8)$ & 10 (13.7) & $9(12.3)$ & $24(32.9)$ & 0.105 \\
\hline Blueberry & $2(2.7)$ & $7(9.6)$ & $4(5.5)$ & $13(17.8)$ & 0.285 \\
\hline Raspberry & - & $2(2.7)$ & $3(4.1)$ & $5(6.8)$ & 0.710 \\
\hline
\end{tabular}

${ }^{*} \mathrm{p}<0.05,{ }^{* *} \mathrm{p}<0.01$,

and storage condition [8]. Moreover, the study was supported by a questionnaire survey among CV patients regarding the effect of certain dietary flavonoids when consumed as part of a normal diet. Out of the 100 invited patients, 73 individuals were participated in the study. The response rate of patients was quite good (73\%). The study showed that the perception and attitude of respondents on diet containing fruits was significantly $(\mathrm{p}<0.05)$ associated with gender, age, and CVDs. The mean age of participants was $59.15 \pm 7.73$ years with minimum age was 42 while the maximum age was 72 years. The majority of the respondents were male $74 \%(n=54)$ while only $26 \%(n=19)$ were female. The demographic data of the participant are illustrated in Table 2.

The result showed that half of the study population $(50.7 \%, n=37)$ had hypertension, whereas $38.4 \%$ prehypertension was noted and merely $11 \%$ of respondents maintained their blood pressure. The study participants were enquired about their preference and consumption of food containing fruits. It was noted that the respondent's attitude toward diet enrich fruits was variable. The study participants preferred and consumed several fruits such as apple, banana, peach, oranges, watermelon, grapefruit, strawberry, blueberry, and raspberry. Even though the majority of the study participants who had either normal blood pressure, prehypertension or hypertension, consumed apples $(83.6 \%)$, oranges $(79.5 \%)$ as well as banana $(64.4 \%)$ respectively $(\mathrm{p}<0.05)$. In addition, the respondents who consumed apple, oranges, and banana had prehypertension $38.4 \%, 37 \%$, and $35.6 \%$, respectively $(p<0.05)$, indicated the lower the risk of hypertension, and can maintain their blood pressure. While the participants who had been taken grapefruit, watermelon, peach and strawberry as a part of their normal diet were also maintained their blood pressure. The responses of the study participants are illustrated in Table 3.

The finding of the present study is consistent with the other epidemiological studies suggested that polyphenolic compound such as quercetin, apigenin, kaempferol, luteolin hesperidin, naringin, and anthocyanin may help to lower the risk of CVD when ingested in large quantities [5]. The reported studies revealed the inverse relations between the flavonoid content and the incidence of mortality for $\mathrm{CV}$ diseases [10]. Consequently, it has been reported that consumption of flavonoid-rich food could decrease the blood pressure and occurrence of CVD. Dietary factors have a substantial role in the regulation of blood pressure as well as lower the risk of blood pressure and their complications [3]. Various Citrus fruits and apple have been a great value for their healthy nutritious and antioxidant properties. Furthermore, the antioxidant and chelating properties of phenolics may protect tissues against oxygen free radicals and lipid peroxidation. It improves the vascular endothelial function, vasodilation, prevents platelet adhesion, increase the HDL and reduced the LDL cholesterol as well as inhibits the smooth muscle proliferation in the vessel wall $[11,12]$. Despite the fact, it is difficult to determine the individual effect of dietary phenolic compounds due to the variability of the genetic factors, gut microflora, sex, age, and lifestyle that may contribute to individual differences in the absorption, distribution, metabolism, and excretion of flavonoids $[4,9,10]$. The findings of the present study also highlighted that respondents are unfamiliar with or skeptical of alternative treatments or beneficial effect of fruits on CVDs as well as other metabolic diseases.

\section{CONCLUSION}

The result of the present study suggested that seasonal fruits have promising health benefit and could be used as a cardiac protective agent. Appropriate awareness and approaches for increased consumption of food enrich with flavonoids should be encouraged as part of a preventive manner against several heart diseases. However, information regarding the effects of flavonoids for the management of hypertension is limited. Therefore, further research is needed to assess the consumer preference for flavonoid consumed foods as well as the quantities required to get their promising health benefits.

\section{ACKNOWLEDGMENT}

The author explicit sincere gratitude to all patients from several hospitals around Karachi, Pakistan.

\section{AUTHORS' CONTRIBUTIONS}

The author declares that all the authors have contributed equally to this article.

\section{CONFLICTS OF INTEREST}

There are no conflicts of interest.

\section{AUTHORS' FUNDING}

The present research work was not funded by any organization or company.

\section{REFERENCES}

1. Mahmoud AM, Bautista RJ, Sandhu MA, Hussein OE. Beneficial effects of citrus flavonoids on cardiovascular and metabolic health. Oxid Med Cell Longev 2019;2019:1-20.

2. Maaliki D, Shaito AA, Pintus G, El-Yazbi A, Eid AH. Flavonoids in hypertension: A brief review of the underlying mechanisms. Curr Opin Pharmacol 2019;45:57-65.

3. Clark JL, Zahradka P, Taylor CG. Efficacy of flavonoids in the management of high blood pressure. Nutr Rev 2015;73:799-822.

4. Mendonça RD, Carvalho NC, Martin-Moreno JM, Pimenta AM, Lopes AC, Gea A, et al. Total polyphenol intake, polyphenol subtypes and incidence of cardiovascular disease: The SUN cohort study. Nutr Metab Cardiovasc Dis 2019;29:69-78.

5. Luo Y, Shang P, Li D. Luteolin: A flavonoid that has multiple cardioprotective effects and its molecular mechanisms. Front Pharmacol 2017;8:1-10.

6. Kokkou E, Bozini N, Lavda M, Mourouzis K, Siasos G. The cardioprotective effects of polyphenols: A focus on classic cardiovascular risk factors. Hell J Atheroscler 2017;8:73-95.

7. Imam S, Azhar I, Perveen S, Hussain SG, Mahmood ZA. Studies on in vitro antioxidant activity and total flavonoid content of a cream formulation to correlate its anti-aging effect. World J Pharm Res 2015;4:1646-55.

8. Aryal S, Baniya MK, Danekhu K, Kunwar P, Gurung R, Koirala N. 
Total phenolic content, flavonoid content and antioxidant potential of wild vegetables from Western Nepal. Plants 2019;8:1-12.

9. Nary FC, Santos RD, Laurinavicius AG, Conceicao RD, Carvalho JA. Relevance of prehypertension as a diagnostic category in asymptomatic adults. Einstein 2013;11:303-9.

10. Ponzo V, Goitre I, Fadda M, Gambino R, De Francesco A, Soldati L, et al. Dietary flavonoid intake and cardiovascular risk: A populationbased cohort study. J Transl Med 2015;13:1-13
11. Dower JI, Geleijinse JM, Gijsbers L, Schalwijk C, Kromhout D, Hollman PC. Supplementation of pure flavonoids epicatechin and quercetin affects some biomarkers of endothelial dysfunction and inflammation in pre hypertensive adults. A randomized double blind, placebo-controlled, cross over trial. J Nutr 2015;145:1459-63.

12. Aptekmann NP, Cesar TB. Long term orange juice consumption is associated with low LDL-cholesterol and apolipoprotein B in normal moderately hypercholesterolemic subjects. Lipid Health Dis 2013;12:1-10. 